\title{
Display Selection of a Hybrid Foldamer-Peptide Macrocycle
}

\author{
Sebastian Dengler, ${ }^{+[a]}$ Ryan T. Howard, ${ }^{+[a]}$ Vasily Morozov, ${ }^{+[a]}$ Christos Tsiamantas, ${ }^{+[b]}$ Zhiwei Liu, ${ }^{[c]}$ \\ Christopher Dobrzanski, ${ }^{[c]}$ Vojislava Pophristic,${ }^{[c]}$ Sophie Brameyer, ${ }^{[d]}$ Céline Douat, ${ }^{[a]}$ Hiroaki Suga, ${ }^{*[b]}$ \\ and Ivan $\mathrm{Huc}^{\star[a]}$
}

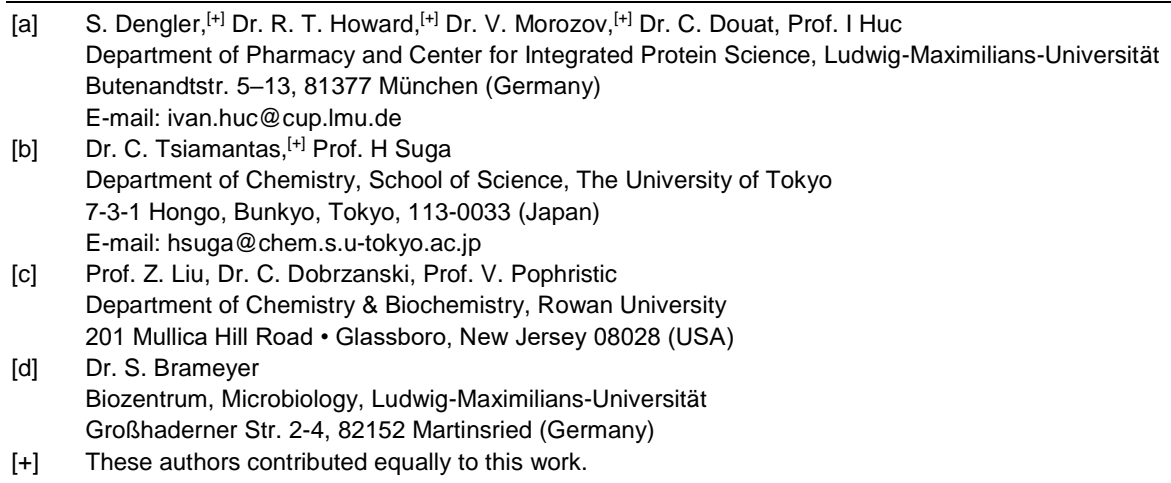

\begin{abstract}
A helical aromatic foldamer was identified that undergoes tRNA acylation by a flexizyme and ribosomal peptide initiation with yields sufficiently high to perform an mRNA display selection of macrocyclic foldamer-peptide hybrids. A hybrid macrocyle binder to the $\mathrm{C}$-lobe of the E6AP HECT domain was selected that showed highly converged peptide residues. A crystal structure and molecular dynamics simulations revealed that both the peptide and foldamer are helical in an intriguing reciprocal stapling fashion. The strong residue convergence could be rationalized based on their involvement in specific interactions. The foldamer stabilizes the peptide helix through stapling and through contacts with key residues. It appears to also contribute to protein binding by direct protein interactions. The results altogether highlight possible benefits in inserting an aromatic foldamer into a peptide macrocycle for the purpose of protein recognition.
\end{abstract}

Peptide and protein display technologies based on nucleic acid sequence encoding are a powerful way to identify potent and selective ligands for biomolecular targets. Such technologies have demonstrated their clinical potential in the development of a range of approved antibody therapies from phage display platforms, ${ }^{[1]}$ and the discovery of peptidic drugs such as ecallantide $^{[2]}$ and romiplostim. ${ }^{[3]}$ Many modern display technologies leverage the incorporation of non-natural modifications into peptide libraries to expand the chemical diversity of these molecules beyond the natural catalogue ${ }^{[4]}$ and allow the discovery of peptides with desirable pharmacological profiles, including resistance to proteolytic degradation, cell permeability, and novel modes of binding. ${ }^{[5-8]}$ Incorporation of these non-natural modifications, e.g. for the purpose of macrocyclization, has been achieved through either postsynthetic modification, ${ }^{[9,10]}$ exploitation of the ribosome's promiscuity, ${ }^{[11,12]}$ or genetic engineering of library host organisms. ${ }^{[13]}$

The remarkable tolerance of the ribosome to accept amino acids with abiotic modifications has recently been highlighted by the incorporation of aromatic oligoamide foldamers (AOFs) into the nascent peptide chain (Figure 1a). Pyridine $(P)$ and quinoline (Q) containing oligomers were charged onto tRNA by flexizymes and ribosomally incorporated into the polypeptide chain affording AOF-peptide hybrids with up to five $\mathrm{P} / \mathrm{Q}$-units as translation initiators, ${ }^{[14,15]}$ or up to three $\mathrm{P} / \mathrm{Q}$-units as sidechain appendages. ${ }^{[16]}$ Functionalization of the $\mathrm{N}$-terminus as a chloroacetamide also allowed for spontaneous macrocyclization with a downstream cysteine thiol. ${ }^{[14,16]}$ The main interest of introducing AOF segments within a peptide macrocycle lies in their inherent folding information. The propensity of $Q_{n}$ and $(P Q)_{n}$ oligomers to adopt stable helical conformations is welldocumented. ${ }^{[17-19]}$ Within a hybrid macrocycle, folding of the helical aromatic segment prevails and alters the peptide conformation, for example by stretching the peptide backbone. ${ }^{[14,20]}$ It also confers resistance towards proteolytic degradation of the peptide. ${ }^{[20]}$ In addition, AOF themselves have potential for directly interacting with proteins ${ }^{[21-27]}$ and they exist as natural products. ${ }^{[28]}$ It was therefore a logical next step to challenge ribosomal translation not just with the production of one AOF-peptide macrocycle, but with an entire DNA-encoded library of such macrocycles. Here we show that FIT-synthesized macrocyclic AOF-peptide hybrids are compatible with mRNA display selection using the random nonstandard peptides integrated discovery (RaPID) platform. Translation initiators containing the foldamer element were optimized for tRNA acylation and translation efficiency, and one initiator was successfully utilized in a selection experiment, generating the first biologically evolved AOF-peptide hybrid macrocycle against a protein target, the catalytic C-lobe of the E6AP HECT domain (Figure S4). ${ }^{[29,30]}$ This E3 ligase was chosen as a proof-of-concept bait protein given its use in a previously successful peptide affinity selection campaign with the RaPID platform. ${ }^{[31]} A$ crystal structure of the macrocycle-protein complex revealed that both the peptide and foldamer are helical in an intriguing reciprocal stapling fashion.

We first set out to identify a foldamer giving sufficiently high yields of both flexizyme-mediated tRNA acylation and ribosomal peptide initiation for the purpose of display selection. $\mathrm{N}$-acylated 
a)

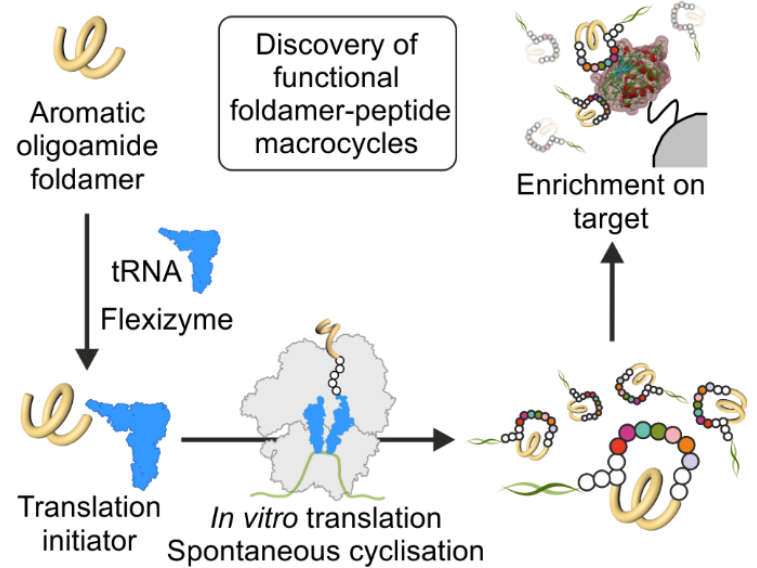

b)

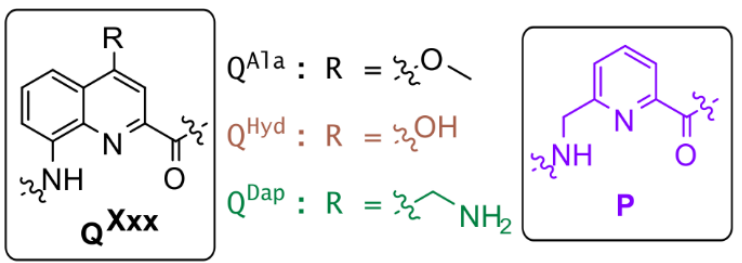

c) 1-6: Ac-Foldamer-GF-CME (CyanoMethyl Ester)

Foldamer $=$

$$
\begin{aligned}
& 1-Q^{A 7 a} P^{A 7 a} \\
& 2 \text {-QDap } P Q A 7 a \\
& 4 \mathrm{PQ}^{\mathrm{Al} a} \mathrm{PQ}^{\mathrm{Ala}} \\
& 5 \mathrm{PQ}^{\mathrm{Dap}} \mathrm{PQ}^{\mathrm{A} 7 \mathrm{a}} \\
& 3-Q^{\text {Hyd }} P Q^{A 7 a} \\
& 6 \mathrm{PQ}^{\mathrm{Hyd}} \mathrm{PQ}^{\mathrm{Ala}} \\
& \text { 7-9: CIAc-Foldamer-GF-CME } \\
& 7 \mathrm{PQ}^{\mathrm{Dap}} \mathrm{PQ}^{\mathrm{Ala}} \\
& 8-Q^{\text {Hyd }} P Q^{A 7 a} \\
& 9 \mathrm{PQ}^{\mathrm{Hyd}} \mathrm{PQ}^{\mathrm{Ala}}
\end{aligned}
$$

Figure 1. a) Schematic of incorporation of aromatic oligoamide foldamers by the flexible in vitro translation (FIT) system into macrocyclic peptide-foldamer hybrids that can undergo selection against a molecular target by mRNA display. b) Nomenclature of the quinoline/pyridine foldamer units used in this study. c) Aromatic foldamer substrates tested for their suitability as translation initiators.

P/Q-based helical oligoamides 1-6 were prepared (Figure 1b,c). The $P$ units serve to introduce some flexibility, ${ }^{[14,32]}$ and side chains on $Q$ units were selected to be either cationic $\left(Q^{\text {Dap }}\right)$, neutral $\left(Q^{\text {Ala }}\right)$, or anionic $\left(Q^{\text {Hyd }}\right)$, though all were kept small to reduce hindrance in the ribosome exit tunnel. The terminal Phe cyanomethyl ester serves as a substrate for the flexizyme. Acylation was assessed using a tRNA mimic, microhelix RNA (mhRNA), demonstrating acylation efficiencies ranging from 0 $85 \%$ (Table 1, Figure S1). Each initiator-charged ${ }^{\text {MMet }}$ tRNA was then supplemented into a Met-deficient in vitro translation system along with an mRNA template. Expression of the desired foldamer-peptide hybrids was demonstrated by MALDI-TOF MS and quantified using autoradiographic gel electrophoresis (Table 1, Figures S2,3). Oligoamides 7-9 were then prepared as $\mathrm{N}$ chloroacetylated $(\mathrm{ClAc})$ analogues of the three top performers $\mathbf{3}$, 5 and 6. tRNA acylation and expression efficiency - including cyclization with a downstream Cys - were determined again (Table 1). The results led to the selection of 7 as an initiator for display selection experiments (Figure 2a,b).

Two independent mRNA display selections were carried out from NNK $(N=A / C / G / T, K=G / T)$ libraries having randomized windows of either 4-9 or 10-15 peptide residues, all including a mandatory Cys and using 7 as the initiator (Figure $2 \mathrm{c}$ ). We a)
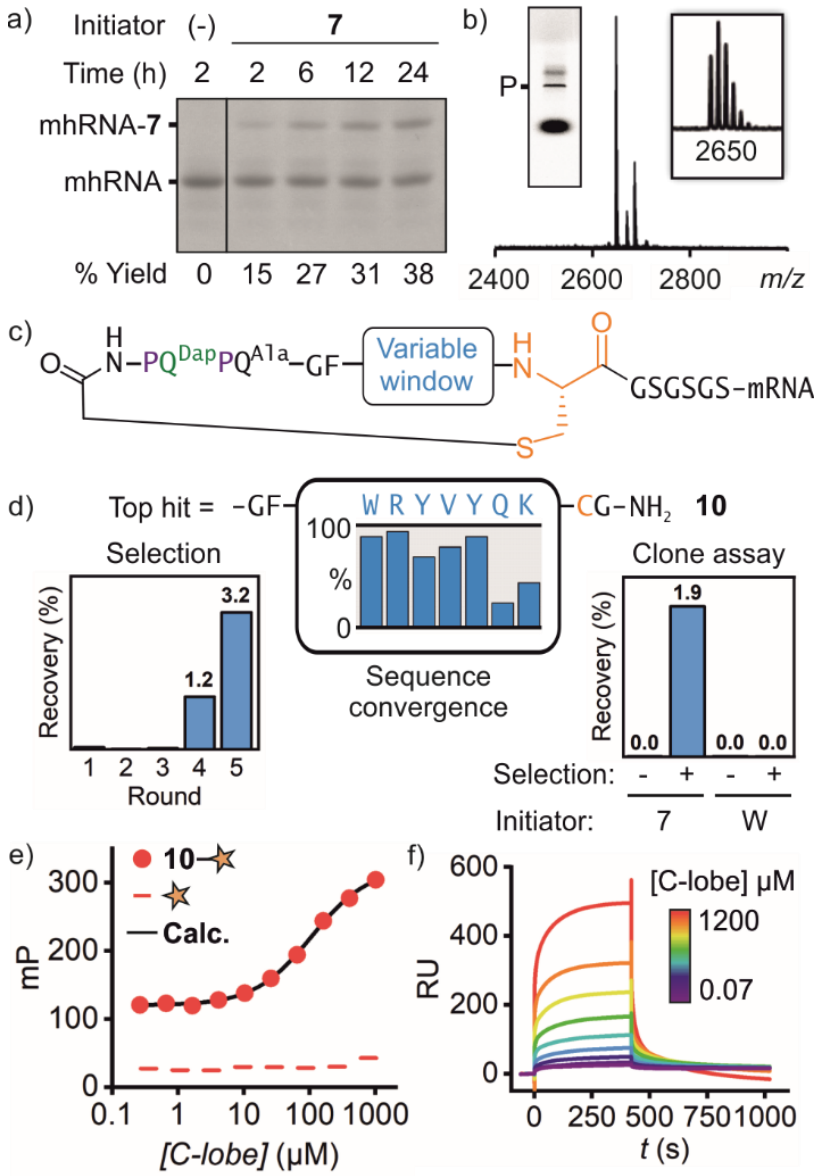

Figure 2. a) Flexizyme-assisted loading of 7 onto truncated tRNA (mhRNA,) assessed by SDS-PAGE; yields were determined by band intensity ratio (mhRNA-7 vs. unmodified mhRNA). b) MALDI MS spectrum of a successfully translated peptide-foldamer macrocycle (see $\mathrm{SI}$ for sequence); right inset = zoom; left inset $=$ quantification of translation $(P, 8 \%$ yield) by radioisotope incorporation. c) General structure of the library of macrocycle-mRNA conjugates. d) Most enriched sequence from the library of smaller macrocycles; sequence conservation after alignment of the top 20 hits is shown; left inset = recovery of DNA after each round of selection as compared to the input library; right inset = recovery of DNA when RaPID selection was performed once with the clonal mRNA of the top hit, using 7 or CIAc-Trp-CME (W) as the initiator, against blank beads (-) or C-lobe modified beads (+); e) Fluorescence polarization of an analogue of $\mathbf{1 0}$ labelled with fluorescein or fluorescein alone (both at $20 \mathrm{nM})$ against C-lobe; data were fit to a 1:1 binding isotherm $\left(K_{d}=107\right.$ $\pm 5 \mu \mathrm{M}$ ). f) SPR sensorgrams of C-lobe (analyte) against a biotin-functionalized analogue of 10 (ligand) on streptavidin chips.

Table 1. Yields of tRNA acylation and peptide translation with foldamer initiators.

\begin{tabular}{cccc}
\hline Initiator & N-terminus & Acylation (\%) & Translation $^{[\mathrm{a}]}(\%)$ \\
\hline $\mathbf{1}$ & Ac & 0 & - \\
$\mathbf{2}$ & Ac & 16 & 5 \\
$\mathbf{3}$ & Ac & 75 & 12 \\
$\mathbf{4}$ & Ac & 14 & $N / D^{[\mathrm{b}]}$ \\
$\mathbf{5}$ & Ac & 85 & 12 \\
$\mathbf{6}$ & Ac & 14 & 13 \\
$\mathbf{7}$ & CIAC & 38 & $8^{[\mathrm{c}]}$ \\
$\mathbf{8}$ & CIAc & 35 & $2^{[\mathrm{c}]}$ \\
$\mathbf{9}$ & CIAc & 67 & $6^{[c]}$ \\
\hline
\end{tabular}

[a] relative to the same reaction with canonical amino acids only (no reprogramming). [b] Smearing did not allow accurate quantification. [c] The template used for quantification is different from that used for other entries. 
surmised that the PQPQ segment would have a larger effect on the structure of shorter peptides, ${ }^{[14,20]}$ but did not know whether this would represent a selection advantage. After five rounds of in vitro selection and amplification, a clear increase in the number of peptides selectively captured by C-lobe-bound magnetic beads could be observed for both libraries (Figure 2d, left, Figure S5), suggesting the emergence of selective binders. Next-generation sequencing of the recovered DNA libraries hinted at an influence of the abiotic segment. The short library converged into a practically unique sequence with a randomized region of 7 amino acid residues (Figure 2d, middle, Figure S6). With the longer library, no such convergence was observed (Figure S6). To assess selectivity for C-lobe and the necessity of the foldamer segment, we conducted independent rounds of selection with only the top mRNA sequence of each library. Challenging the translation products of these sequences against beads with and without C-lobe (clone assay) resulted in the binding and recovery of material only when C-lobe was present, suggesting binding of these sequences to the target (Figure 2d right, Figure S7). When the experiment was conducted substituting the foldamer- ${ }^{\mathrm{IM} M \text { t }} \mathrm{tRNA}$ initiator with CIAc-Trp- ${ }^{\mathrm{fMet}} \mathrm{tRNA}$, binding of the shorter peptide macrocycle was abolished, highlighting a direct or indirect, but essential role of the foldamer in the binding interactions. The effect was lower in the case of the peptide from the larger macrocycle library. Given these results, further studies focused on a hit from the enriched species of smaller macrocycle library.

Foldamer-peptide macrocycle $\mathbf{1 0}$ (Figure 2d), an analogue of the top hit from library $\mathrm{NNK}^{4-9}$, was then chemically synthesized first by automated solid-phase synthesis of the peptide element, followed by automated solid-phase foldamer synthesis to install the $P Q^{\text {Dap }} P Q^{A l a}$ tetramer. Subsequent manual incorporation of the chloroacetamide was performed on-resin before cleavage and deprotection, thioether macrocyclization, and purification by reverse-phase HPLC. The ${ }^{1} \mathrm{H}$ NMR spectrum of $\mathbf{1 0}$ exhibited characteristics of a helically-folded foldamer component (Figure S8). ${ }^{[20]}$ The circular dichroism (CD) spectrum of $\mathbf{1 0}$ was flat in the quinoline absorption region (300-400 nm, Figure S8) indicating no preference for either $P$ or $M$ foldamer helicity. In contrast, peptide chirality was shown to bias foldamer helix handedness in PQPQpeptide macrocycles with shorter peptide loops. ${ }^{[14,20]}$ Upon adding C-lobe to 10, the CD spectrum at $300-400 \mathrm{~nm}$ remained flat (Figure S9), unlike with other foldamers with which proteinmediated handedness bias was observed. ${ }^{[21,22]}$ This indicates that, if any binding occurs, the $P$ and $M$ conformers of $\mathbf{1 0}$ bind equally well. The binding of $\mathbf{1 0}$ to C-lobe was demonstrated using biotinylated and fluorescein-labelled derivatives (Scheme S1). A triplicate fluorescence polarization binding study revealed a $K_{d}=$ $107 \mu \mathrm{M}$ (Figure 2e). No concomitant binding was observed against the fluorophore alone, and the macrocycle did not engage lysozyme, a protein of similar size and secondary structure composition as C-lobe (Figure S10). Orthogonal binding assessment by surface plasmon resonance (SPR) was undertaken. While association and dissociation events were too slow to fit their respective rate constants from the sensorgrams, plotting the maximum response against concentration generated a $K_{d}$ similarly in the high $\mu \mathrm{M}$ range (Figure $2 f$, Figure S11).

Binding was not as high as hoped and too low to make biological studies, e.g. inhibition, worthy of interest. Nevertheless, the high convergence of the selected sequence hinted at selective macrocycle/C-lobe interactions (Figure 2d). The complex was thus crystallized and its structure in the solid state was solved in space group $C 2$ at $2.3 \AA$ resolution (Figures $\mathrm{S} 12-19$ ). The asymmetric unit contains two molecules of $\mathbf{1 0}$ and four of $\mathrm{C}$-lobe. The protein has the same structure as the $\mathrm{C}$-lobe of an intact

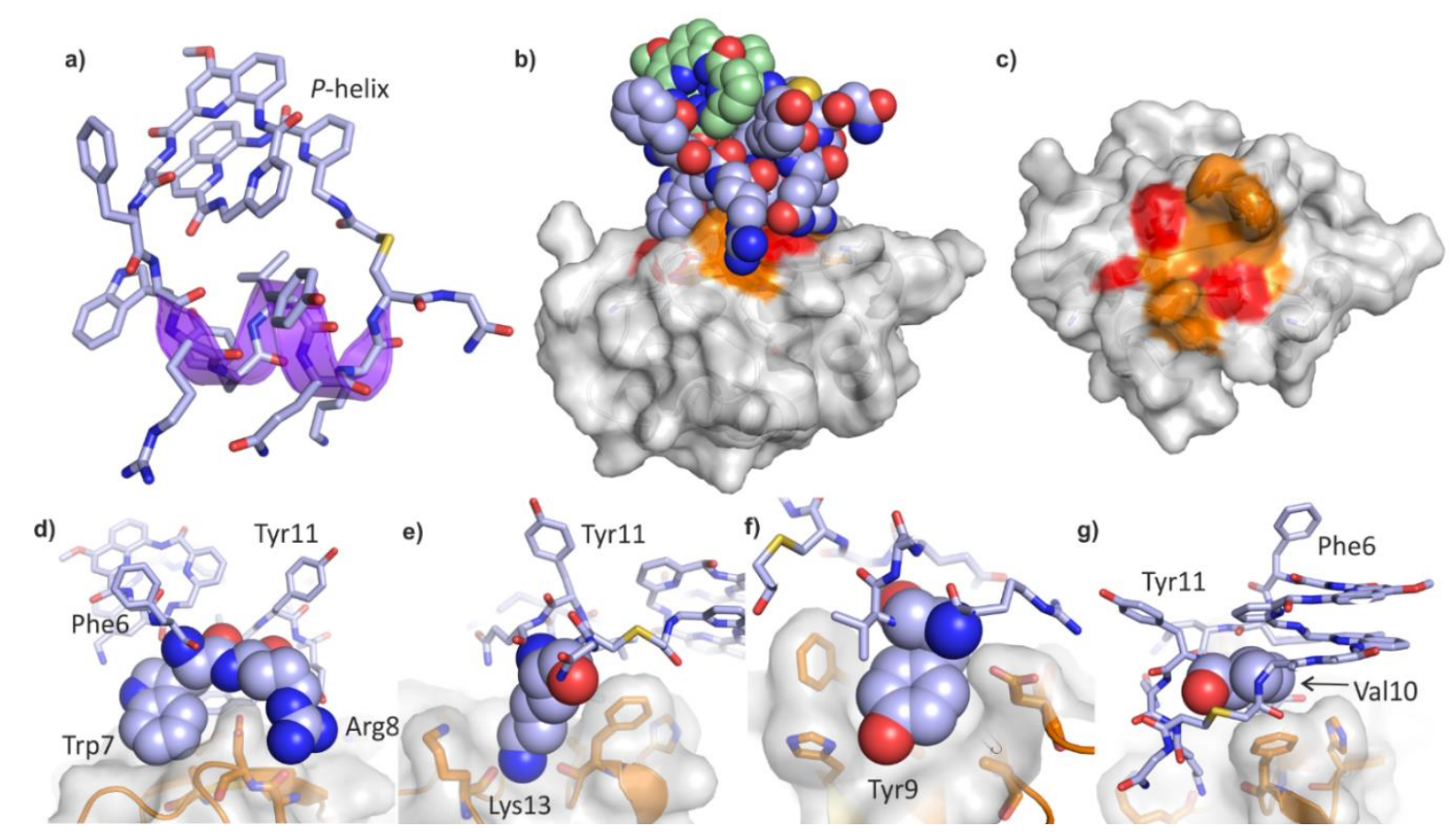

Figure 3. Crystal structure of the complex between C-lobe and macrocycle $\mathbf{1 0}$ (PDB \#7QPB). a) View of the macrocycle alone showing the peptide and foldamer helices. b) Overall view of the complex. Macrocycle $\mathbf{1 0}$ is shown in space filling representation. Carbon atoms in green belong to the foldamer. c) Top view of the macrocycle binding site on the protein. Hydrogen bonds, salt-bridges, and hydrophobic contacts are highlighted in orange and longer distance hydrophobic contacts are shown in red. $\mathrm{d}-\mathrm{g}$ ) Specific macrocycle protein contacts. Residues of interest are shown in space filling representation. Residues of $\mathbf{1 0}$ are numbered starting from the N-terminus of the foldamer segment (P1, Q2, P3, Q4, Gly5, Phe6... Cys14, Gly15). 
E6AP HECT domain whose coordinates were used for molecular replacement (PDB \#1C4Z). ${ }^{[29]}$ Domain swapping dimerization between two molecules of $C$-lobe was not observed. ${ }^{[30]}$ However, careful examination revealed that the four proteins are in fact two disulfide-bridged dimers (Figure S15). Oxidation of Cys820 presumably occurred during crystallization.

The macrocycle was found in a remarkable conformation in which both the peptide and foldamer segments are helically folded (Figure 3a, Figure S16), as if stapling each other. The peptide forms a slightly distorted $\alpha$-helix, e.g. with some bifurcated hydrogen bonds (Figure S17). With only eight residues involved (WRYVYQKC), it would not be expected to be stable without an intramolecular staple. ${ }^{[33]}$ The PQPQ helix matches well with earlier structures. ${ }^{[14,20]}$ In contrast with its conformation in solution, the foldamer helix is exclusively $P$ in the solid state, indicating induction of handedness during crystallization. Crystal packing is relatively tight; as shown in detail in the supporting information, each macrocycle $\mathbf{1 0}$ makes contacts with four distinct proteins (Figures S12-15, S18-19). However, most of the peptide residues that converged during display selection, i.e. the $\alpha$-helix, form an extended and intimate binding area with one of these four proteins (Figure $3 b, c$ ), whereas other contacts are limited and mostly concern the peptide residues that were not selected and one $Q$ unit of the foldamer. It can therefore be proposed that the largest contact area - ca $400 \AA^{2}$ - constitutes the actual binding region. The proximity between the binding region and the disulfide bridge make it impossible for two macrocycles to simultaneously bind a C-lobe disulfide dimer (Figure S15), shedding light on the 2:1 Clobe-macrocycle stoichiometry in the crystal.

The protein-macrocycle interface of the complex involves extended hydrophobic contacts (with Gly755, Gly756, Ala805, Ile 803, Phe821, Val823), salt bridges and hydrogen bonds (Figure S18). Several of the highly converged peptide residues show high shape and interaction complementarity with the protein surface, notably Trp7, Arg8, Tyr9, Val10 and Lys13 (Figure 3d-g). The most converged residue, Arg8, is clamped by Asp754 and Glu752 of C-lobe. Val10 occupies the core of the macrocycle and is in contact with both C-lobe and the foldamer. The crystal structure thus largely explains sequence convergence during selections, but it leaves two questions open: how the macrocycle with $M$ foldamer helicity binds as well as with $P$ helicity (as shown by the absence of induced CD) and why Tyr11 displays high selection convergence yet it points away from the cognate C-lobe in the crystal structure.

MD simulations were used to tentatively answer these questions (Figure 4, Figure S19-27). The complex found in the solid state was used as a starting point of MD runs. The complex underwent little change for some time before exploring other binding modes. In particular, direct binding of the foldamer to the hydrophobic protein area was observed while the $\alpha$-helix was still folded but exposed to water (Figure 4d). MD runs of a V10A mutant showed a destabilization of the $\alpha$-helix (Figure S27, Table S2), highlighting the structural contribution of Val10. A model of the macrocycle with an $M$ foldamer helix was then built (Figure 4a) and energy minimized in complex with C-lobe while keeping the $\alpha$-helix essentially intact. MD runs showed that the complex was kept with the same interactions and at least for as long as with the $P$ helix. The macrocycle with the $M$ helix then also explored alternate binding modes involving extensive foldamerprotein contacts (Figure 4c). A notable feature was the tight and persistent stacking of Tyr11 on the foldamer, at the core of the macrocycle (Figure 4b), in a role similar to that of Val10 with the $P$ foldamer helix that may explain the selection of Tyr11 as a stabilizer of the $\alpha$-helix with the $M$ helical foldamer. Conversely, with the $M$ foldamer helix, the V10A mutation did not result in a collapse of the $\alpha$-helix (Figure S26).
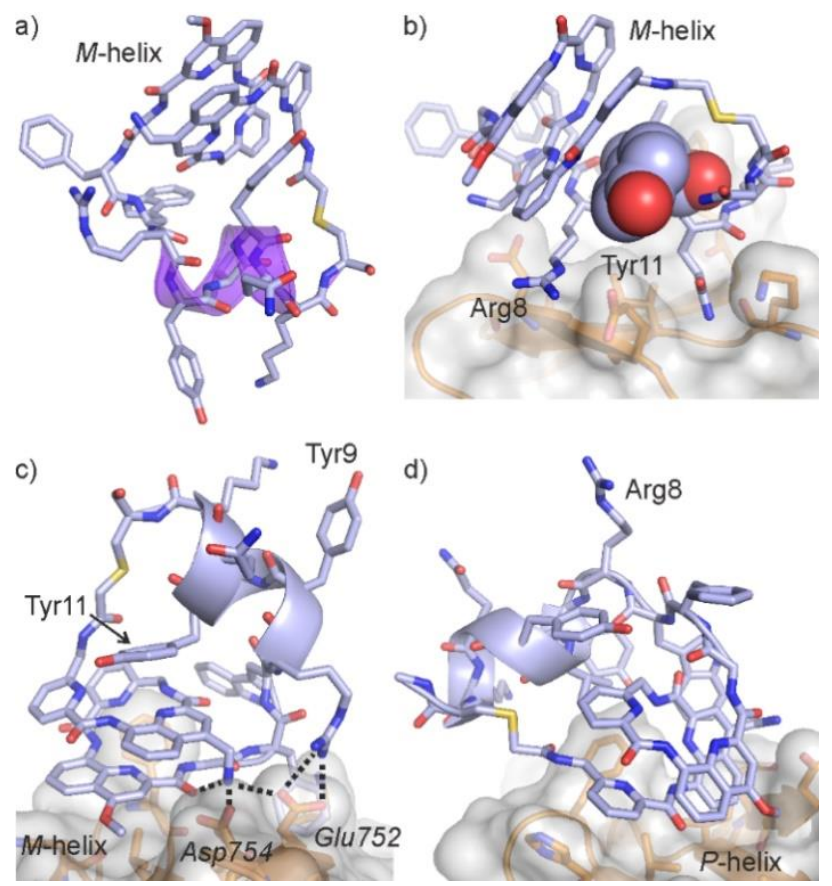

Figure 4. Representative snapshots of MD simulations of $\mathbf{1 0}$ in complex with $\mathrm{C}$ lobe. A) View of $M$-helical 10 (the protein has been removed for clarity). B) Binding of $M$-helical $10 \mathrm{C}$-lobe in a mode similar to that of the crystal structure. C) Alternate binding mode of $M$-helical $\mathbf{1 0}$ showing extensive protein-foldamer contacts. D) Same as c) but with $P$-helical 10. The alpha-helix of $\mathbf{1 0}$ is shown as a solid ribbon in a), c) and d). Some remarkable hydrogen bonds are shown as dashed lines.

In conclusion, following a successful display selection of a macrocyclic foldamer-peptide hybrid, a crystallographic structure and MD simulations highlighted the possible roles of the residues that converged most during selection. The foldamer appears to stabilize the peptide $\alpha$-helix not only by firmly holding its two ends but also via critical peptide residue-foldamer contacts. MD simulations also suggest that binding modes exist other than that found in the solid state in which the foldamer establishes direct contacts with the protein. New selection experiments against other protein targets are in progress to further explore the possible benefits of introducing a foldamer helix within a peptide macrocycle.

\section{Acknowledgements}

This work was supported by the DFG (SFB1309 and HU1766/21) to I.H. and the JSPS Grant-in-Aid for Specially Promoted Research (JP21H05618) to H.S. We thank Dr. Sunbum Kwon for assistance with chemical synthesis and Dr. Sonia Lorenz for providing the expression vector for C-lobe. We are grateful to the European Synchrotron Radiation Facility (ESRF, Grenoble, France) for providing the beamtime (proposal $m \times 2279$ ), and Local 
Contact at the ESRF for providing assistance in using beamline ID23-1. ${ }^{[34]}$ We thank University of the Sciences and West Pharmaceutical Services, Inc. for providing computational resources used for MD simulations.

Keywords: Foldamer $\cdot$ Macrocycle $・$ Peptide $・$ Display selection -Protein recognition

[1] R. Barderas, E. Benito-Peña, Anal. Bioanal. Chem. 2019, 411, 24752479.

[2] J. A. Bernstein, M. Qazi, Expert Rev. Clin. Immunol. 2010, 6, 29-39.

[3] G. Molineux, A. Newland, Br. J. Haematol. 2010, 150, 9-20.

[4] M. Muttenthaler, G. F. King, D. J. Adams, P. F. Alewood, Nat. Rev. Drug Discov. 2021, 20, 309-325.

[5] X.-D. Kong, J. Moriya, V. Carle, F. Pojer, L. A. Abriata, K. Deyle, C. Heinis, Nat. Biomed. Eng. 2020, 4, 560-571.

[6] S. Imanishi, T. Katoh, Y. Yin, M. Yamada, M. Kawai, H. Suga, J. Am. Chem. Soc. 2021, 143, 5680-5684.

[7] Y. V Guillen Schlippe, M. C. T. Hartman, K. Josephson, J. W. Szostak, J. Am. Chem. Soc. 2012, 134, 10469-10477.

[8] C. Tsiamantas, M. E. Otero-Ramirez, H. Suga, Methods Mol. Biol. 2019, 2001, 299-315.

[9] C. A. Rhodes, D. Pei, Chem. - A Eur. J. 2017, 23, 12690-12703.

[10] H. Tsutsumi, T. Kuroda, H. Kimura, Y. Goto, H. Suga, Nat. Commun. 2021, 12, 696 .

[11] T. Katoh, K. Tajima, H. Suga, Cell Chem. Biol. 2017, 24, 46-54

[12] T. Kawakami, H. Murakami, H. Suga, Chem. Biol. 2008, 15, 32-42.

[13] B. Oller-Salvia, J. W. Chin, Angew. Chem. Int. Ed. 2019, 58, 10844 10848; Angew. Chem. 2019, 131, 10960-10964.

[14] J. M. Rogers, S. Kwon, S. J. Dawson, P. K. Mandal, H. Suga, I. Huc, Nat. Chem. 2018, 10, 405-412.

[15] C. Tsiamantas, S. Kwon, C. Douat, I. Huc, H. Suga, Chem. Commun. 2019, 55, 7366-7369.

[16] C. Tsiamantas, S. Kwon, J. M. Rogers, C. Douat, I. Huc, H. Suga, Angew. Chem. Int. Ed. 2020, 59, 4860-4864; Angew. Chem. 2020, 132, 48904894.

[17] H. Jiang, J.-M. Léger, I. Huc, J. Am. Chem. Soc. 2003, 125, 3448-3449.

[18] M. Kudo, V. Maurizot, B. Kauffmann, A. Tanatani, I. Huc, J. Am. Chem. Soc. 2013, 135, 9628-9631.

[19] B. Baptiste, C. Douat-Casassus, K. Laxmi-Reddy, F. Godde, I. Huc, J. Org. Chem. 2010, 75, 7175-7185.

[20] S. Dengler, P. K. Mandal, L. Allmendinger, C. Douat, I. Huc, Chem. Sci. 2021, 12, 11004-11012.

[21] M. Jewginski, T. Granier, B. Langlois d'Estaintot, L. Fischer, C. D. Mackereth, I. Huc, J. Am. Chem. Soc. 2017, 139, 2928-2931.

[22] M. Vallade, M. Jewginski, L. Fischer, J. Buratto, K. Bathany, J.-M. Schmitter, M. Stupfel, F. Godde, C. D. Mackereth, I. Huc, Bioconjug. Chem. 2019, 30, 54-62.

[23] K. Ziach, C. Chollet, V. Parissi, P. Prabhakaran, M. Marchivie, V. Corvaglia, P. P. Bose, K. Laxmi-Reddy, F. Godde, J.-M. Schmitter, S. Chaignepain, P. Pourquier, I. Huc, Nat. Chem. 2018, 10, 511-518.

[24] Z. Hegedus, C. M. Grison, J. A. Miles, S. Rodriguez-Marin, S. L. Warriner, M. E. Webb, A. J. Wilson, Chem. Sci. 2019, 10, 3956-3962.

[25] V. Azzarito, J. A. Miles, J. Fisher, T. A. Edwards, S. L. Warriner, A. J. Wilson, Chem. Sci. 2015, 6, 2434-2443.

[26] S. Kumar, M. Birol, D. E. Schlamadinger, S. P. Wojcik, E. Rhoades, A. D. Miranker, Nat. Commun. 2016, 7, 11412.

[27] S. Kumar, A. D. Hamilton, J. Am. Chem. Soc. 2017, 139, 5744-5755.

[28] T. Seedorf, A. Kirschning, D. Solga, Chem. - A Eur. J. 2021, 27, 73217339.

[29] L. Huang, E. Kinnucan, G. Wang, S. Beaudenon, P. M. Howley, J. M. Huibregtse, N. P. Pavletich, Science 1999, 286, 1321-1326.

[30] L. K. Ries, A. K. L. Liess, C. G. Feiler, D. E. Spratt, E. D. Lowe, S. Lorenz, Protein Sci. 2020, 29, 1550-1554.

[31] Y. Yamagishi, I. Shoji, S. Miyagawa, T. Kawakami, T. Katoh, Y. Goto, H. Suga, Chem. Biol. 2011, 18, 1562-1570.

[32] M. Vallade, P. Sai Reddy, L. Fischer, I. Huc, Eur. J. Org. Chem. 2018,
2018, 5489-5498

[33] R. S. Harrison, N. E. Shepherd, H. N. Hoang, G. Ruiz-Gómez, T. A. Hill, R. W. Driver, V. S. Desai, P. R. Young, G. Abbenante, D. P. Fairlie, Proc. Natl. Acad. Sci. 2010, 107, 11686-11691.

[34] D. Nurizzo, T. Mairs, M. Guijarro, V. Rey, J. Meyer, P. Fajardo, J. Chavanne, J.-C. Biasci, S. McSweeney, E. Mitchell, J. Synchrotron Rad. 2006, 13, 227-238. 